\title{
Are recent Arctic ozone losses caused by increasing greenhouse gases?
}

\author{
Harald E. Rieder ${ }^{1,2}$ and Lorenzo M. Polvani ${ }^{1,2,3}$ \\ Received 25 June 2013; revised 1 August 2013; accepted 6 August 2013; published 27 August 2013.
}

[1] It has been suggested that the Arctic ozone losses observed in recent years might be a manifestation of climate change due to increasing greenhouse gases. We here offer evidence to the contrary, by focusing on the volume of polar stratospheric clouds ( $\left.V_{\mathrm{PSC}}\right)$, a convenient proxy for polar ozone loss whose simplicity allows for easily reproducible results. First, we analyze the time series of $V_{\mathrm{PSC}}$ in three reanalysis data sets and find no statistically significant trends in $V_{\mathrm{PSC}}$-nor changes in their probability density functions-over the period 1979-2011. Second, we analyze $V_{\text {PSC }}$ in a stratosphere-resolving chemistry-climate model forced uniquely with increasing greenhouse gases following the A1B scenario: here too, we find no significant changes in $V_{\text {PSC }}$ over the entire 21 st century. Taken together, these results strongly suggest that the sporadic high ozone losses in recent years have not been caused by increasing greenhouse gases. Citation: Rieder, H. E., and L. M. Polvani (2013), Are recent Arctic ozone losses caused by increasing greenhouse gases?, Geophys. Res. Lett., 40, 4437-4441, doi:10.1002/grl.50835.

\section{Introduction}

[2] It is well established that man-made ozone-depleting substances (ODS) are the primary cause of stratospheric ozone depletion [Molina and Rowland, 1974; Rowland and Molina, 1975]. Since the detection of the Antarctic ozone hole in the 1980s [e.g., Farman et al., 1985], the evolution of the Earth's ozone layer, especially at high latitudes, has received much attention. Notably, the Montreal Protocol and its amendments have led to a significant decline in the stratospheric burden of ODS [World Meteorological Organization (WMO), 2011], and the decline in ODS has had a measurable effect on column ozone [Mäder et al., 2010; WMO, 2011]. Furthermore, state-of-the-art chemistryclimate models consistently project ozone levels to recover to pre-1980 values in the second half of the 21 st century [e.g., SPARC-CCMVal, 2010].

[3] In light of this, the Arctic ozone losses during winter 2010/2011 came somewhat unexpected [Manney et al., 2011,

Additional supporting information may be found in the online version of this article.

${ }^{1}$ Lamont-Doherty Earth Observatory, Columbia University, Palisades, New York, USA.

${ }^{2}$ Department of Applied Physics and Applied Mathematics, Columbia University, New York, New York, USA.

${ }^{3}$ Department of Earth and Environmental Sciences, Columbia University, New York, New York, USA.

Corresponding author: H. E. Rieder, Lamont-Doherty Earth Observatory, Columbia University, Palisades, NY 10964, USA. (hrieder@1deo.columbia.edu)

(C)2013. American Geophysical Union. All Rights Reserved. 0094-8276/13/10.1002/grl.50835 hereinafter M11], although previous WMO/UNEP (United Nations Environment Programme) Ozone Assessment Reports highlighted the possibility of severe Arctic ozone depletion in particularly cold winters. The 2010/2011 event, referred to as the "first Arctic ozone hole" [e.g., Garcia, 2011; Sinnhuber et al., 2011; M11], has received much attention in the literature, with several studies providing a highly detailed picture of the chemical and dynamical state of the Arctic atmosphere during that winter [e.g., Adams et al., 2012; Lindenmaier et al., 2012; Pommerau et al., 2013; Sinnhuber et al., 2011; M11]. One key question, however, remains unanswered: Are such extreme Arctic ozone losses, which appear to have occurred more frequently in recent decades, a manifestation of climate change due to increasing greenhouse gases?

[4] The aim of this paper is to answer this question. We do so by studying the evolution of lower stratospheric temperatures: these are crucial to the formation of polar stratospheric clouds (PSCs) which are of central importance for stratospheric ozone depletion in polar winter [e.g., Peter, 1997; Solomon, 1999]. In particular, the volume of air that is colder than the threshold for PSC formation (hereinafter $V_{\mathrm{PSC}}$ ) is a widely used and convenient proxy for polar ozone depletion [Rex et al., 2004, hereinafter R04]. In the last several decades, an upward trend in the evolution of $V_{\text {PSC }}$ has been reported $[W M O, 2011]$, with a new record approximately every 5 years, leading to the popular notion that "the coldest Arctic winters are getting colder" [e.g., Rex et al., 2006, hereinafter R06], possibly as a consequence of increasing greenhouse gases. But is this really so?

[5] In this paper, we carefully analyze $V_{\mathrm{PSC}}$ in three different reanalysis data sets and find no statistically significant changes in recent decades. We also analyze the output of a chemistry-climate model forced uniquely with increasing greenhouse gases from 1960 to 2100 and again find no trends in $V_{\mathrm{PSC}}$ over that entire period. From these facts, we conclude that it is premature to interpret recent large Arctic ozone losses as a signal of Arctic climate change induced by increasing greenhouse gases.

\section{Data and Methods}

[6] We follow the original approach of R04 and compute $V_{\text {PSC }}$ using daily temperatures at 30 and $50 \mathrm{hPa}$, from three reanalysis data sets: NASA-Modern Era Retrospective Analysis for Research and Applications (NASA-MERRA) [e.g., Rienecker et al., 2011], European Centre for MediumRange Weather Forecasts (ECMWF) ERA-Interim [Dee et al., 2011], and National Centers for Environmental Prediction-National Center for Atmospheric Research (NCEP-NCAR) [e.g., Kalnay et al., 1996; Kistler, 2001]. Note that these reanalyses have different horizontal resolutions: $0.5^{\circ} \times 0.66^{\circ}$ for NASA-MERRA, $1.5^{\circ} \times 1.5^{\circ}$ for ERA-Interim, and $2.5^{\circ} \times 2.5^{\circ}$ for NCEP-NCAR. 

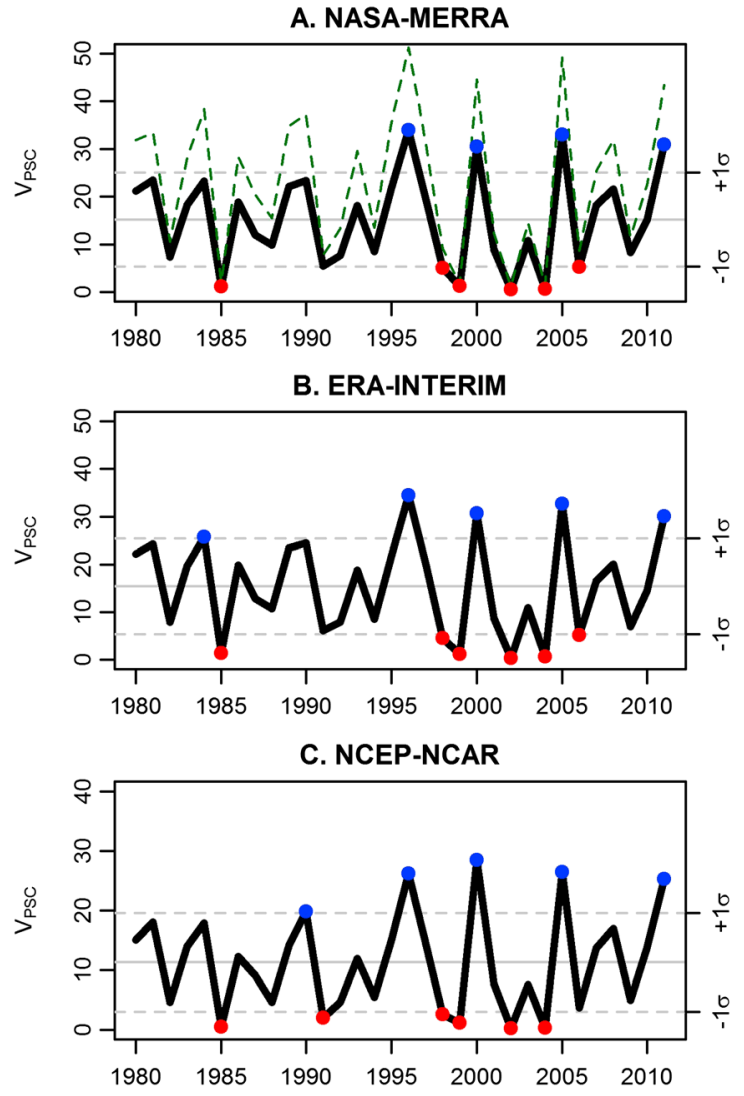

Figure 1. Black lines represent extended wintertime $V_{\text {PSC }}\left(10^{6} \mathrm{~km}^{3}\right)$ in $1979 / 1980-2010 / 2011$ for the (a) NASAMERRA, (b) ERA-Interim, and (c) NCEP-NCAR reanalysis data sets. Blue (red) points represent the years where $V_{\mathrm{PSC}}$ is outside of plus (minus) 1 standard deviation (dotted grey lines) of the 1979/1980-2010/2011 mean (solid grey lines). The green curve in Figure 1a represents unscaled $V_{\mathrm{PSC}}$ from M11.

[7] For each day, $V_{\mathrm{PSC}}$ is computed by first identifying the grid cells north of $60^{\circ} \mathrm{N}$ whose temperatures $T$ fall below the critical threshold for nitric acid trihydrate (NAT) formation. Following Hanson and Mauersberger [1988], we use $T_{\mathrm{NAT}}=193.61 \mathrm{~K}$ at $30 \mathrm{hPa}$ and $T_{\mathrm{NAT}}=195.59 \mathrm{~K}$ at $50 \mathrm{hPa}$. Next, we calculate the total PSC area $\left(A_{\text {PSC }}\right)$ by summing the areas of all grid cells with $T<T_{\mathrm{NAT}}$. Finally, following R04, we obtain $V_{\text {PSC }}$ from $A_{\text {PSC }}$ using the expression

$$
\begin{aligned}
V_{\mathrm{PSC}}= & {\left[0.8 \times A_{\mathrm{PSC}}(50 \mathrm{hPa}) \mathrm{km}^{2}+0.2 \times A_{\mathrm{PSC}}(30 \mathrm{hPa}) \mathrm{km}^{2}\right] } \\
& \times 5.06 \mathrm{~km}
\end{aligned}
$$

[8] From these daily values, we obtain the mean value of $V_{\text {PSC }}$ for each Arctic winter by averaging over the extended season 16 December to 15 April. To test robustness, we have also computed $V_{\mathrm{PSC}}$ for the shorter season 16 December to 31 March and found no significant differences.

[9] For the modeling component of this study, daily values of $T$ were not available. However, daily $A_{\text {PSC }}$ was archived, albeit only at $50 \mathrm{hPa}$. In view of this, we use the simpler expression

$$
V_{\mathrm{PSC}} *=\left[0.8 \times A_{\mathrm{PSC}}(50 \mathrm{hPa}) \mathrm{km}^{2}\right] \times 5.06 \mathrm{~km}
$$

[10] We are aware that this simplification affects the absolute magnitude of $V_{\mathrm{PSC}}$; however, since $A_{\mathrm{PSC}}$ at $50 \mathrm{hPa}$ is multiplied by a much larger coefficient (see equation (1) above), this simplification does not affect the overall pattern or robustness of the results. In fact, we have compared $V_{\mathrm{PSC}}$ and $V_{\mathrm{PSC}^{*}}$ in the NASA-MERRA reanalysis: Figure S1a in the supporting information shows that the two quantities are very tightly related, the latter being consistently smaller, on average, by $25 \%$. We also note that in a number of recent studies [e.g., Tilmes et al., 2006; M11], $V_{\text {PSC }}$ was further scaled by the volume of the polar vortex. Here we decided to analyze unscaled $V_{\mathrm{PSC}}$ as there are many possible ways to define the vortex, and such scaling adds, in our view, unnecessary complexity to the simple formulation given in equation (1). In fact, we have compared scaled and unscaled $V_{\text {PSC }}$ for NASA-MERRA and found that the correlation is very high (0.92).

\section{Evidence From Reanalyses}

[11] The time series of extended winter time $V_{\mathrm{PSC}}$, which was computed using equation (1) over the period 1979/ 1980-2010/2011, is shown in Figure 1 for each of the three data sets we have analyzed. Contrasting the abscissa on the individual panels, one can see that the actual magnitude of $V_{\text {PSC }}$ varies considerably among the three reanalyses, with NASA-MERRA and ERA-Interim being roughly 30\% larger than NCEP-NCAR. Such differences have been documented before and are directly related to the individual temperature biases in the data sets. Manney et al. [2003], for instance, contrasted six different reanalyses and found that the areas of low temperatures in the lower stratosphere can differ by as much as $50 \%$ among different products, with NCEPNCAR, in general, being the warmest, as confirmed in Figure 1. Hence, NCEP-NCAR should be used with much caution in detailed polar process studies.

[12] More importantly, however, Figure 1 reveals a remarkable agreement in the year-to-year variability of $V_{\mathrm{PSC}}$ among the three reanalysis data sets. The correlations among the three time series in Figure 1 are extremely high, ranging from 0.95 (between ERA-Interim and NCEP-NCAR) to 0.99 (for NASA-MERRA and ERA-Interim). This strong agreement among different data sets, combined with the extreme simplicity of the underlying method used here for computing $V_{\text {PSC }}$, makes our results very robust.

[13] The first result to be gathered from Figure 1 is that no trend in $V_{\mathrm{PSC}}$ is immediately discernible over the 32 year period for which reliable data are available from multiple data sets. To confirm this, we have computed trends in several different ways and found nothing that is statistically significant on a rigorous test level (i.e., 95\%). Specifically, we have computed the trend over (1) the entire time series, (2) the first and second halves separately, and (3) 5 year and 3 year maximum and minimum values. The $p$ values for all of these are reported in Table S1: no trends are significant on a 95\% level.

[14] Next, we focus on the "extreme" years, as these have received the most attention in the previous literature. These years are the key piece of evidence behind the suggestion that "the coldest winters are getting colder" [e.g., R06]. We start by objectively defining the "coldest winters" as those for which the $V_{\text {PSC }}$ values are more than 1 standard deviation above the mean (computed over each of the 1979/ 1980-2010/2011 time series). These coldest winters are 

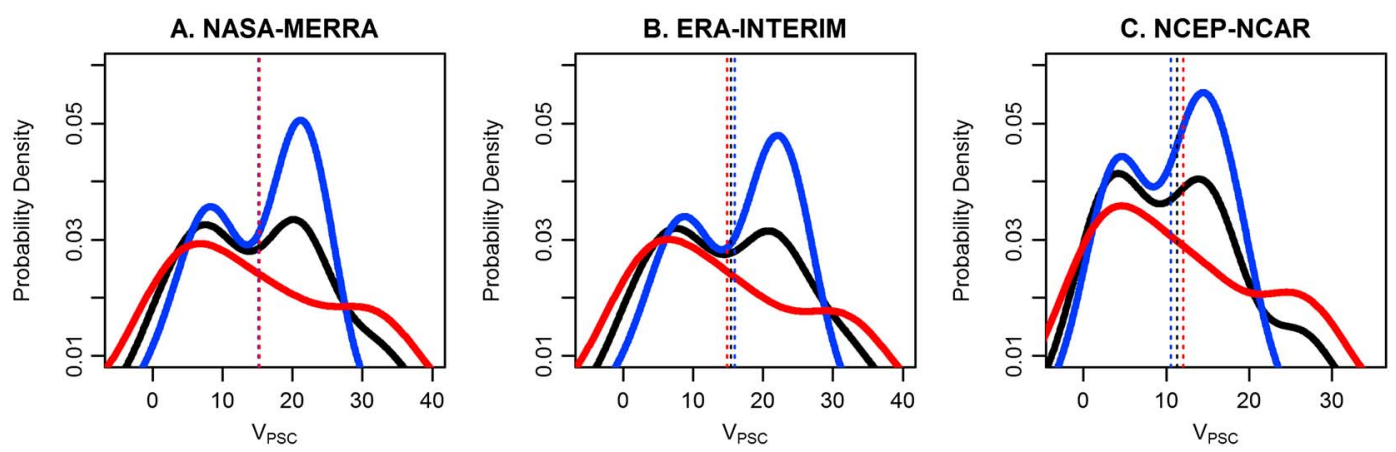

Figure 2. Probability density plots of extended wintertime $V_{\mathrm{PSC}}\left(10^{6} \mathrm{~km}^{3}\right)$ for the 1979/1980-2010/2011 (black), 1979/19801994/1995 (blue), and 1995/1996-2010/2011 (red) time periods as computed from the (a) NASA-MERRA, (b) ERA-Interim, and (c) NCEP-NCAR reanalysis data sets. The colored dotted lines mark the respective mean values of the individual time periods. Probability densities are calculated using the "density" function with a "Gaussian" kernel from the standard "stats" package in the statistical software R. The statistical properties of the kernel $(K)$ are determined by $\sigma^{2}(K)=\operatorname{int}\left(t^{2} K(t) \mathrm{d} t\right)$, which is always equal to 1 (and hence the bandwidth is the standard deviation of the kernel) and $\mathrm{R}(K)=\operatorname{int}\left(K^{2}(t) \mathrm{d} t\right)$.

highlighted, for each reanalysis, by the blue dots in Figure 1. It is abundantly clear that no visible positive trends exist in these coldest winters, in any of the reanalyses here presented. It seems that extremely cold winters are more frequent in the second half of the record (see below), but note that the actual ranking of the coldest winter is not robust across the reanalyses. For instance, the winter of 1999/2000 is the coldest in NCEP-NCAR (Figure 1c), but it is clearly not the coldest in the other two reanalyses. More importantly, we see no evidence for a steady and monotonic increase in $V_{\text {PSC }}$ for the coldest winters, such as, e.g., the one shown in the 2010 Ozone Assessment Report [WMO, 2011, Figure 2-16] or in R04 and R06. The explanation for this major qualitative difference, we suggest, lies in the fact that while our $V_{\text {PSC }}$ time series originate from individual, and therefore internally consistent, reanalysis products, the earlier studies have merged possibly inconsistent data sets. Specifically, the $V_{\text {PSC }}$ time series analyzed in R04 and R06 were constructed by combining radiosonde data for the presatellite era with ECMWF operational analyses after 1979 . We note that the latter alone have undergone significant changes in the assimilation scheme and the number of assimilation levels in 1997 and again in 1999 [Pommerau et al., 2013]. Furthermore, Figure 1 shows that not only the coldest winters but also the warmest winters (red dots) appear to be more frequent in the second half of the record. If indeed the coldest winters are getting colder as a consequence of climate change induced by increasing greenhouse gases, the naive expectation would be that the entire stratosphere would be cooling, and thus, the warmest winters should be less frequent in recent decades: Figure 1 testifies to the contrary.

[15] Although robust trends are clearly absent from the time series in Figure 1, the fact remains that the majority of extreme winters-both cold and warm-are found in the second half of the record, and this occurs robustly across all three reanalyses. The visual impression one gets from Figure 1 is that perhaps, a regime shift occurred around 1995 , so that extreme winters have become more frequent since. In order to test whether this impression is in fact statistically significant, we carefully compare the first and second halves of the time series.

[16] Specifically, in Figure 2, we show the probability density functions of $V_{\text {PSC }}$ for the entire time period (black), the first half of the record (1979/1980-1994/1995, blue), and the second half (1995/1996-2010/2011, red); for clarity, each reanalysis is shown in a different panel. The blue and red curves appear quite different, at first glance. To determine if this difference is statistically significant, we apply two standard statistical tests. First, we use a Kolmogorov-Smirnov (KS) test, the standard nonparametric test for the equality of continuous one-dimensional probability distributions. The results of the KS test are that the probability distributions do not differ significantly from each other in any of the three reanalysis data sets (all $p$ values $>0.7$ ). Second, we use a more conservative test that assigns greater weight to the tails. A $k$-sample Anderson-Darling (ADK) test, a standard rank test without restrictive parametric model assumptions for the equality of continuous one-dimensional probability distributions [e.g., Scholz and Stephens, 1987], confirms the results of the KS test. Given the highly insignificant $p$ values of the KS and ADK tests, we conclude that the samples in the first and second halves of the record are stemming from the same overall probability distribution. In simpler terms, events found after 1995 could have occurred before 1995 with equal probability, and vice versa. This result also holds if different (and shorter) time windows are compared (not shown here).

[17] We conclude this section by emphasizing that our results are highly insensitive to the precise definition of $V_{\text {PSC }}$. For instance, M11 used seven isotropic surfaces in the lower stratosphere $(390,410,430,460,490,520$, and $550 \mathrm{~K}$ ) to calculate $V_{\text {PSC }}$. One might ask whether such complexity is needed. The green dashed line in Figure 1a shows the $V_{\text {PSC }}$ computed by M11: while the absolute numbers are consistently higher than the ones obtained from the original R04 definition (as one would expect from simply adding more vertical levels), the correlation between the green and black lines is very high (a value of 0.98 , as illustrated in Figure S1b).

\section{Evidence From Chemistry-Climate Model Integrations}

[18] Having demonstrated that reanalyses show no evidence that "the coldest winters are getting colder" over the last 32 years, we now turn to analyzing model output. It is conceivable that the observational record is simply too short, but a clear climate signal would appear on a longer time scale 

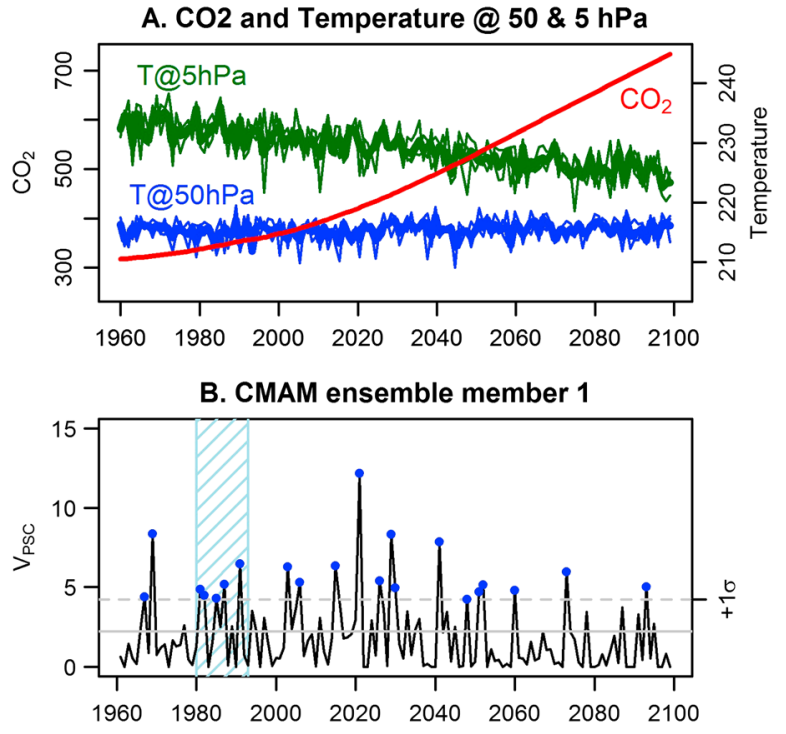

C. CMAM ensemble member 2

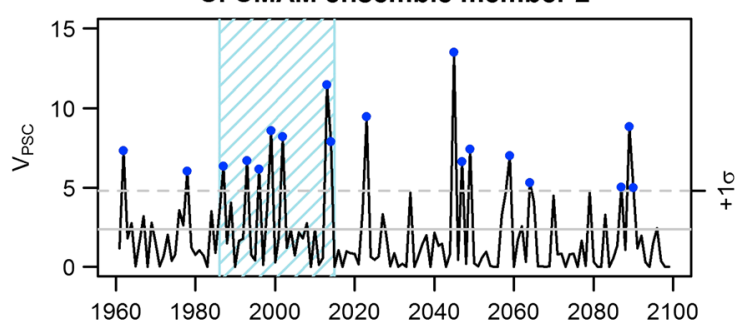

D. CMAM ensemble member 3

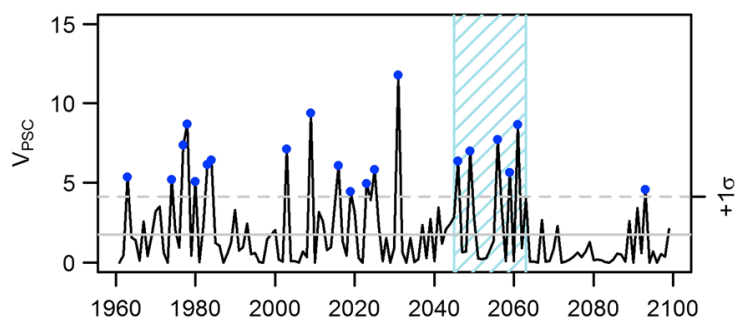

Figure 3. (a) Annual mean $\mathrm{CO}_{2}$ concentration (ppm) (red) and mean wintertime (December-April) polar cap $\left(60^{\circ} \mathrm{N}-\right.$ $90^{\circ} \mathrm{N}$ ) temperatures $(\mathrm{K})$ at the 50 (blue) and 5 (green) $\mathrm{hPa}$ levels, from the Canadian Middle Atmosphere Model (CMAM) ensemble under the SCN-B2b scenario (bold lines show ensemble averages; thin lines show individual ensemble members). (b-d) Extended wintertime $V_{\mathrm{PSC}} *\left(10^{6} \mathrm{~km}^{3}\right)$ (black solid line) from 1960/1961 to $2099 / 2100$ in the three CMAM ensemble members under the SCN-B2b scenario. Blue points mark the years where $V_{\mathrm{PSC}^{*}}$ is outside of plus 1 standard deviation (dotted grey lines) of the 1979/1980 2010/2011 mean value (solid grey lines). Blue hashed boxes highlight periods with increasing patterns in $V_{\mathrm{PSC}} *$ extremes.

with a larger greenhouse gas forcing. In the present context, what is needed are model runs with increasing greenhouse gases alone, to see if the coldest winters do indeed become colder (in the lower stratosphere) as a consequence of such an increase.

[19] As it happens, such runs were suggested as part of the Chemistry-Climate Model Validation Activity 2 (CCMVal-2) and were referred to as the "SCN-B2b scenario": high-top models with interactive stratospheric chemistry were to be integrated from 1960 to 2100 with only well-mixed greenhouse gases increasing as per the SRES A1B scenario, but with all other forcings kept fixed at 1960 levels [SPARC-CCMVal, 2010]. Unfortunately, only few such runs actually are available (as they were not made mandatory in CCMVal-2); for clarity of presentation, we focus here on an ensemble of three SCN-B2b runs performed with the Canadian Middle Atmosphere Model (CMAM), the only model for which an ensemble of such runs was available. The CMAM model and its SCN-B2b runs have been extensively documented [McLandress et al., 2010, 2011] in the context of the ozone hole in the Southern Hemisphere. Here we shift the focus to the Arctic lower stratosphere and how it changes as a consequence of increasing greenhouse gases, keeping in mind that CMAM was also the only CCMVal-2 model with a fully coupled ocean, thus simulating the surface warming due to the greenhouse effect in an internally consistent way (all other CCMVal-2 models were run with specified sea surface temperatures).

[20] To set the stage, the red line in Figure $3 \mathrm{a}$ shows the $\mathrm{CO}_{2}$ concentrations in these runs: note that the values more than double from 1960 to 2100 . As a consequence of the increasing $\mathrm{CO}_{2}$, the upper stratosphere cools, as one expects: this is illustrated by the green curve in the same figure, showing the $60^{\circ} \mathrm{N}-90^{\circ} \mathrm{N}$ polar cap temperature at $5 \mathrm{hPa}$, averaged over the three ensemble members (the individual members are the thin curves of the same color). However, the lower stratosphere is little affected by the increasing greenhouse gases: see how the blue curve, showing the lower stratospheric polar cap temperature at $50 \mathrm{hPa}$, is nearly flat. This is not a peculiar behavior of the CMAM model. It is well established that greenhouse gas cooling manifests itself primarily in the middle and upper stratosphere and is very small in the lower stratosphere; see, for instance, the classic study of Manabe and Wetherald [1967, Figure 16]. Furthermore, using a comprehensive general circulation model, Sigmond et al. [2004] explored the effects of $\mathrm{CO}_{2}$ doubling on lower/middle stratospheric temperature and showed that the very small signal results from a cancellation between radiative cooling at upper levels and tropospheric dynamical heating.

[21] Since the lower stratospheric, polar cap, mean temperature carries no signature of the increasing greenhouse gases in the CMAM integrations, it is not surprising that the extreme temperatures show no trends as well (as documented for an earlier version of CMAM) [Hitchcock et al., 2009]. In Figures $3 b-3 d$, we plot the time series of $V_{\text {PSC }} *$ for each of the three CMAM ensemble members from 1960 to 2100 . The blue dots show the coldest winters, i.e., those which fall outside 1 standard deviation of the 1979/1980-2010/2011 mean, as in Figure 1. There are clearly no meaningful trends in the blue dots, over the entire 140 year period. Nonetheless, one can easily identify multidecadal periods in which a sequence of coldest winters (blue dots) appears to be getting colder: these are highlighted with the blue hashing, to emphasize the fact that such periods are entirely fortuitous in these model runs.

\section{Conclusion}

[22] Over the 32 year period 1979-2011, three widely used reanalysis data sets show no trends in the volume of polar stratospheric clouds $\left(V_{\mathrm{PSC}}\right)$, when computed in a simple 
and reproducible way from daily temperatures at two lower stratospheric levels. In addition, although high- $V_{\text {PSC }}$ years appear to cluster after 1995, we find that the probability density distributions are not statistically different before and after that year. These findings are in agreement with the recent study of Pommerau et al. [2013], who document the absence of statistically significant trends in the amplitude of denitrification in early winter, renoxification of the vortex, vortex strength, or total ozone depletion, over the period 1994 2011. Furthermore, analyzing the output from a model forced solely with increasing greenhouse gases, we find no evidence of a temperature trend in the lower stratosphere, although $\mathrm{CO}_{2}$ concentrations more than double over the length of those model runs. While these results come from a single model, they offer compelling evidence that greenhouse gases are unlikely to have a major effect on lower stratospheric temperatures in Arctic spring.

[23] Acknowledgments. H.E.R acknowledges the support of the Swiss National Science Foundation (SNF) through fellowship gran PEZP2-134426. L.M.P. was partly supported by a grant from the U.S. National Science Foundation. The authors are grateful to Gloria Manney, Rolf Mueller, Thomas Peter, Tiffany Shaw, Ted Shepherd, and Susan Solomon for enlightening discussions on this topic. The authors also thank Gloria Manney and Zac Lawrence for $A_{\text {PSC }}$ data from the NASA-MERRA Reanalysis and Charles McLandress for providing the CMAM data. The authors thank Markus Rex for detecting a plotting mistake in Figure 1c in an early draft of this paper.

[24] The Editor thanks Jean-Pierre Pommereau and an anonymous reviewer for their assistance in evaluating this paper.

\section{References}

Adams, C., et al. (2012), Severe 2011 ozone depletion assessed with 11 years of ozone, $\mathrm{NO}_{2}$, and $\mathrm{OClO}$ measurements at 80 degrees N, Geophys. Res. Lett., 39, L05806, doi:10.1029/2011GL050478.

Dee, D. P., et al. (2011), The ERA-Interim reanalysis: Configuration and performance of the data assimilation system, Q. J. R. Meteorol. Soc., 137(656), 553-597.

Farman, J. C., et al. (1985), Large losses of total ozone in Antarctica reveal seasonal CLOX/NOX interaction, Nature, 315(6016), 207-210.

Garcia, R. R. (2011), Atmospheric science, An Arctic ozone hole?, Nature, 478(7370), 462-463.

Hanson, D., and K. Mauersberger (1988), Laboratory studies of the nitricacid trihydrate-Implications for the south polar atmosphere, Geophys. Res. Lett., 15(8), 855-858.

Hitchcock, P., et al. (2009), Past and future conditions for polar stratospheric cloud formation simulated by the Canadian Middle Atmosphere Model, Atmos. Chem. Phys., 9(2), 483-495.

Kalnay, E., et al. (1996), The NCEP/NCAR 40-year reanalysis project, Bull. Am. Meteorol. Soc., 77(3), 437-471.
Kistler, R. (2001), The NCEP-NCAR 50-year reanalysis: Monthly means CD-ROM and documentation, Bull. Am. Meteorol. Soc., 82, 247-268.

Lindenmaier, R., et al. (2012), Unusually low ozone, $\mathrm{HCl}$, and $\mathrm{HNO}_{3}$ column measurements at Eureka, Canada during winter/spring 2011, Atmos. Chem. Phys., 12(8), 3821-3835.

Mäder, J. A., et al. (2010), Evidence for the effectiveness of the Montreal Protocol to protect the ozone layer, Atmos. Chem. Phys., 10, 12,161-12,171.

Manabe, S., and R. Wetherald (1967), Thermal equilibrium of atmosphere with a given distribution of relative humidity, J. Atmos. Sci., 24(3), 241-259.

Manney, G. L., et al. (2003), Lower stratospheric temperature differences between meteorological analyses in two cold Arctic winters and their impact on polar processing studies, J. Geophys. Res., 108(D5), 8328, doi:10.1029/2001JD001149.

Manney, G. L., et al. (2011), Unprecedented Arctic ozone loss in 2011, Nature, 478(7370), 469-475.

McLandress, C., et al. (2010), Separating the dynamical effects of climate change and ozone depletion. Part I: Southern hemisphere stratosphere, J. Clim., 23(18), 5002-5020.

McLandress, C., et al. (2011), Separating the dynamical effects of climate change and ozone depletion. Part II: Southern hemisphere troposphere, J. Clim., 24(6), 1850-1868.

Molina, M. J., and F. S. Rowland (1974), Stratospheric sink for chlorofluoromethanes-Chlorine atomic-catalysed destruction of ozone, Nature, 249(5460), 810-812.

Peter, T. (1997), Microphysics and heterogeneous chemistry of polar stratospheric clouds, Annu. Rev. Phys. Chem., 48, 785-822.

Pommerau, J. P., et al. (2013), Why unprecedented ozone loss in the Arctic in 2011? Is it related to climatic change?, Atmos. Chem. Phys., 13, 5299-5308.

Rex, M., et al. (2004), Arctic ozone loss and climate change, Geophys. Res. Lett., 31, L04116, doi:10.1029/2003GL018844.

Rex, M., et al. (2006), Arctic winter 2005: Implications for stratospheric ozone loss and climate change, Geophys. Res. Lett., 33, L23808, doi:10.1029/2006GL026731.

Rienecker, M. M., et al. (2011), MERRA: NASA's Modern-Era Retrospective Analysis for Research and Applications, J. Clim., 24(14), 3624-3648.

Rowland, F. S., and M. J. Molina (1975), Chlorofluoromethanes in environment, Rev. Geophys., 13(1), 1-35.

Scholz, F. W., and M. A. Stephens (1987), $K$-sample Anderson-Darling tests, J. Am. Stat. Assoc., 82(399), 918-924.

Sigmond, M., et al. (2004), A simulation of the separate climate effects of middle-atmospheric and tropospheric $\mathrm{CO}(2)$ doubling, J. Clim., 17(12), 2352-2367.

Sinnhuber, B. M., et al. (2011), Arctic winter 2010/2011 at the brink of an ozone hole, Geophys. Res. Lett., 38, L24814, doi:10.1029/2011GL049784.

Solomon, S. (1999), Stratospheric ozone depletion: A review of concepts and history, Rev. Geophys., 37(3), 275-316.

SPARC-CCMVal (2010), SPARC Report on the Evaluation of ChemistryClimate Models, edited by V. Eyring, T. G. Shepherd, and D. W. Waugh, SPARC Report No. 5, WCRP-132, WMO/TD-No. 1526.

Tilmes, S., et al. (2006), Chemical ozone loss in the Arctic and Antarctic stratosphere between 1992 and 2005, Geophys. Res. Lett., 33, L20812, doi:10.1029/2006GL026925.

World Meteorological Organization (WMO) (2011), Scientific Assessment of Ozone Depletion: 2010, Global Ozone Research and Monitoring Project-Report No. 52, 516 pp., Geneva, Switzerland. 